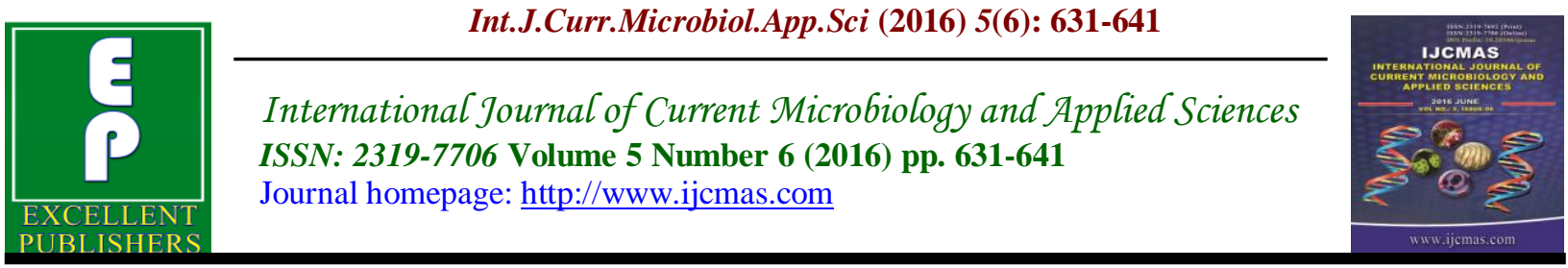

Original Research Article

http://dx.doi.org/10.20546/ijcmas.2016.506.069

\title{
Prevalence and Risk Factors for Post Cesarean Delivery Surgical Site Infection in a Teaching Hospital Setting in Rural Rwanda: A Prospective Cross Sectional Study
}

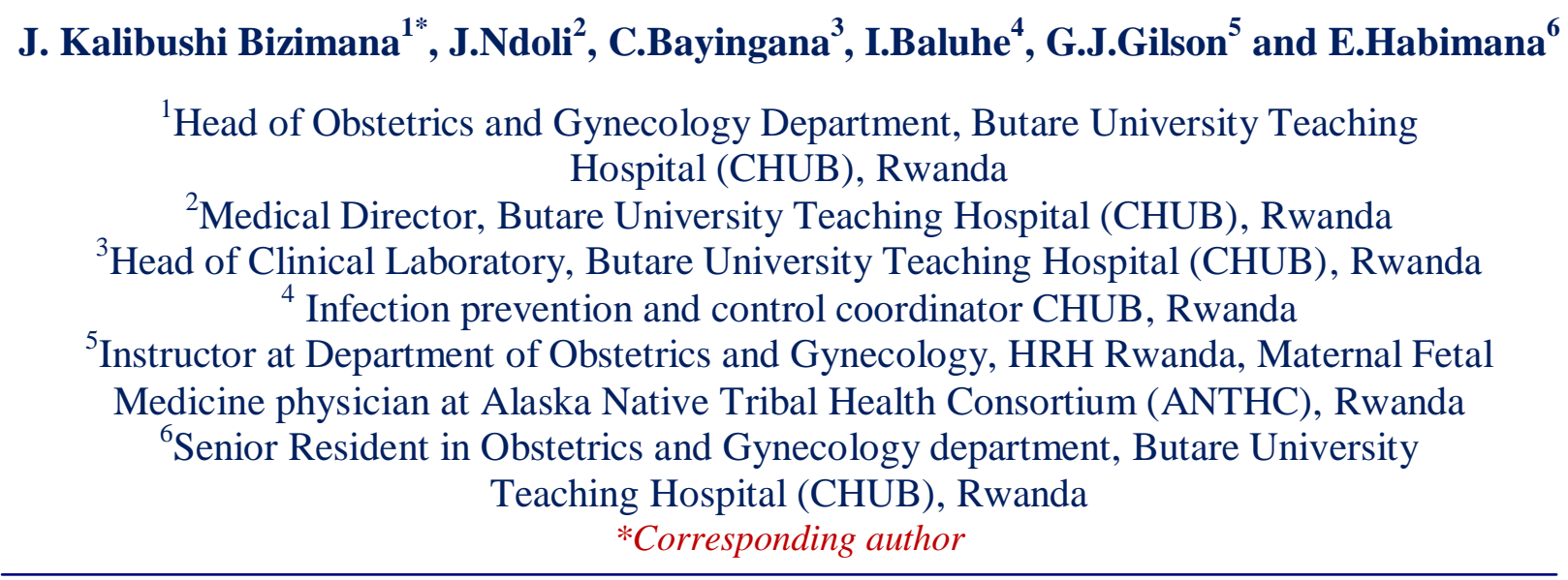

A B S T R A C T

\section{Keywords}

Surgical site infection, cesarean delivery, risk factors, post discharge surveillance.

\section{Article Info}

Accepted:

23 May 2016 Available Online:

10 June 2016
Cesarean delivery (CD) is one of the commonest surgeries worldwide. Surgical site infection (SSI) is one of the complications which arises later on in the postoperative period with an incidence five to twenty times that of vaginal delivery. Knowing the prevalence of SSIs and its risk factors is important in improving infection prevention and control in our setting. This study aimed to assess the prevalence and risk factors for post CD SSI in the department of Gynecology and Obstetrics ofButare University Teaching Hospital (CHUB). This is a prospective cross sectional study, where women who had CD at CHUB during the study period were followed up for duration of thirty days. Before discharging them from the hospital, all SSI signs and symptoms were discussed. The surgical sites were inspected once on discharge day and the dressing was removed. Data was collected using a questionnaire which was filled out by the study nurse. A telephone interview was carried out in two occasions at $15^{\text {th }}$ and $30^{\text {th }}$ day after discharge inquiring about SSI symptoms and signs. Were also instructed to call anytime if she had any signs or symptoms of infection. A total of 323 women who had CD were recruited and followed up for 30 days. No participants were lost during the follow up. They were 16 total SSI for a prevalence of $4.9 \%$. The majority of infections were superficial surgical site infection (75\%) and most of the infections developed after discharged (62.5\%). There were 2 deep SSI and 2 organ SSI and no infection related maternal deaths. Significant association was found between prolonged labor and SSI development. No significant association was noted with duration of surgery, rupture of membranes and obesity. Elective CD (53.6\%) comprised the majority of operation. Staphylococcus aureus (62.5\%) was the predominant organism isolate in microbiological studies. Subcuticular suture was the most skin type closure without increasing risk of SSI. Prophylactic antibiotics and Abdominal and thigh pre preparation for surgery with Alcohol firstly and secondly povidone were used in $100 \%$ of our study population. The prevalence of SSI at CHUB was found to be high at $4.9 \%$ on women undergoing $\mathrm{CD}$ and prolonged labor was found to be significantly associated risk of developing SSI. Use of mobile telephone after proper explanation of signs and symptoms of SSI to women underwent CD was a best post discharge tools for SSI evaluation. 


\section{Introduction}

Cesarean delivery is the term commonly used to describe the delivery of an infant through an abdominal uterine incision. Cesarean delivery has played a major role in lowering both maternal and perinatal morbidity and mortality rates during the past century. The initial purpose of the operation was to preserve the life of the mother with obstructed labor, but indications expanded over the years to include delivery for a variety of more subtle dangers to the mother or fetus. Contributing to its more frequent use is increased safety that is largely due to better surgical technique, improved anesthesia, effective antibiotics, and availability of blood transfusion. (Danforth's Obstetrics and gynecology, 2008) But surgical site infection (SSI) is one of the complications which arise later on in the postoperative period

The incidence of cesarean delivery (CD) has increased exponentially; making it the most commonly performed surgical procedure worldwide. While modern anesthesia, blood banking, and antibiotics have generally made it a safe procedure, surgical site infection (SSI) is a definite, and often serious, maternal complication. Classical risk factors for SSI are multiple and include: preexisting infection, low serum albumin, older age, smoking, diabetes mellitus, obesity, ischemia due to vascular disease or irradiation, prolonged procedure and inadequacies in either the surgical scrub or the antiseptic preparation of the skin. Other factors related to the skill of the surgeon such as poor surgical techniques, inadequate hemostasis, and the presence of dead space, predispose to greater incidence of wound infection.

Surgical site infections are associated with substantial morbidity and mortality, prolonged hospital stay, and increased cost. Obstetrical surgery involves some degree of contamination, and is classified" cleancontaminated" cases, even when the patient has no preoperative symptoms of active infection. Pregnant women are at risk of infection during labor and delivery, which may be enhanced by the postulated relative immunocompromised state of gestation. (CDC, 2013; John Larsen, 2003) The widespread use of antibiotic prophylaxis has reduced but not eliminated the serious postoperative infections; the average expected incidence of SSI being 3-15\% after cesarean delivery. These rates are increased in presence of other risk factors such as gross contamination of the operative site, prolonged and premature rupture of membranes, prolonged and premature rupture of membranes, prolonged operative time, obstructed labor, obesity, chorioamnionitis, emergency operation and altered immune status, which is especially common in poor countries where women may be malnourished and chronically anemic. (Raka, 2006)

In developing countries, especially in sub Saharan Africa, the average wound infection rates are twice or three times higher developed countries.

In Ethiopia, the overall SSI rate was reported to be $21 \%$ in the general surgical wards of teaching hospitals.

In Rwanda, reported postoperative infection rates from 40 district hospitals were $0.8 \%$, $0.4 \%$ and $0.2 \%$ for 2008, 2009 and 2010 respectively (Petroze et al., 2011) these numbers are suspected to be underestimated, as follow up may have described elsewhere. However no study has been done so far to assess the prevalence and risk factors of post cesarean delivery surgical site infection in large referral teaching hospitals in our 
country, such as Butare University Teaching Hospital. (CHUB)

SSI are sometimes remarked in CHUB but appropriate antibiotic prophylaxis, proper surgical technique, adequate hemostasis and surgical timing have decreased significantly the rate of post cesarean delivery surgical site infection.

The principal objectives of this study were to assess the prevalence and risk factors for post cesarean delivery surgical site infection in Butare University Teaching Hospital Ob/Gyn Department. But the specific ones were to determine the epidemiological profile of post cesarean SSI and the major clinical signs and symptoms those facilitate the rapid diagnosis of post cesarean SSI in CHUB, identify risk factors of post cesarean SSI in CHUB, evaluate the management of post cesarean SSI in CHUB and elaborate preventive measures of post cesarean SSI in CHUB.

\section{Methods}

The study was conducted in the Department of Gynecology and Obstetrics at Butare University Teaching Hospital (CHUB) which is a referral and teaching hospital in the southern province of Rwanda. Recruitment was done in obstetrical ward and post cesarean delivery ward. The Department was constituted by admission room, labor ward, immediate postpartum ward, late postpartum ward, Emergency room, operating rooms, recovery room, post gynecological surgery ward, obstetrical ward, postoperative infection ward, outpatient department, oncology service and family planning

A prospective cross section study design was used to follow recruited parturient undergoing cesarean delivery for up to 30 days post-surgery.Data collection was done from $1^{\text {st }}$ January 2015 to $31^{\text {st }}$ July 2015 by using a structured questionnaire and follow up sheet. Recruitment included all patients underwent elective or emergency cesarean section at Butare University Teaching Hospital (CHUB) during the study period. After consenting for participation in the study the women were followed for 30 days in postoperative period, or longer if needed. Women were excluded if the participant died before completion of surveillance sheet post cesarean delivery.

Surgical site infection (SSI) was identified during hospital stay or within thirty days following cesarean section by readmission to the hospital or by post discharge survey, using a combination of telephone calls at 15and 30 day by the study coordination or health worker questionnaires. Surgical site infection (SSI) was diagnosed based on the criteria of the Center for Diseases Control and Prevention (CDC).

Standard definitions of SSI recognized that it affects the following parts of the operative site:

-Superficial incision: involves only the skin (wound cellulitis) or the subcutaneous tissue (wound abscess) of the incision that occurs within 30 days of surgery.

-Deep incision: involves the deep tissues facial and muscle layers (i.e. Necrotizing myofasciitis).

-Organ/space infection: involves any part of the anatomy, other than the incision, opened or manipulated during the surgical procedure (CDC 1992). Following cesarean delivery this most commonly occurs as endomyometritis, but may also occur as intraabdominal abscess, generalized peritonitis, or septic pelvic vein 
thrombophlebitis. (CDC, 2013)

* Criteria for superficial incisional infection: 1. Purulent drainage from the superficial incision

2. The superficial incision yields organisms from the culture of aseptically aspiration fluid or tissue, or from the swab and pus cells are present.

3. At least two of the following symptoms and signs: Pain or tenderness, localized swelling, redness, heat and the superficial incision is deliberately opened by a surgeon to manage the infection, unless the incision is culture-negative. Or b. the clinician diagnoses a superficial incisional infection (wound cellulitis).

Note: Stitch abscesses are defined as minimal inflammation and discharge confined to the points of suture penetration, and localized infection around a stab wound. They are not classified as surgical site infections.

*Criteria for deep incisional infection

1. Purulent drainage from the deep incision but not from the organ/space component of surgical

site.

2. The deep incision yields organisms from the culture of aseptically aspirated fluid or tissue, or from a swab and pus cells are present.

3. A deep incision that spontaneously dehisces or deliberately opened by surgeon when the patient has at least one of the following symptoms or signs (unless the incision is culture-negative): -Fever $\left(>38^{\circ} \mathrm{C}\right)$, -Localized pain or tenderness.
4. An abscess or other evidence of infection involving the deep incision that is found by direct examination during re operation, or by histopathological or radiological examination.

5. Diagnosis of deep incisional surgical site infection by an attending clinician.

Note: An infection involving both superficial and deep incision is classified as deep incisional SSI unless there are organisms present at each site.

*Criteria for organ/space infection:

1. Purulent drainage from a drain that is placed through a stab wound into the organ/space

2. The organ/space yields organisms from the culture of aseptically aspirated fluid or tissue, or from a swab and pus cells are present.

3. An abscess or other evidence of infection involving the organ/space that is found by direct examination, or by histopathological or radiological examination.

4. Diagnosis of an organ/space infection by an attending clinician.

Participants were reviewed during hospital stay by using a cesarean section surveillance data sheet which was completed in theatre room. Wound observation was done on the post cesarean delivery ward, on re admission, or in the outpatient department. Socio demographic characteristics, cesarean delivery category, indications for operation, duration of labor, duration of rupture of membranes, duration of surgery, type of surgical site infections, estimated blood loss, American Society of Anesthesiologists (ASA) Risk assessment tool, Body Mass Index (BMI), hospital stay duration, type of 
sutures and surgeon grade, were recorded. All participants suspected for having SSI, had the wound swab cultured and antibiotic sensitivity was done if the wound swab was positive.

A total of 323 participants were recruited based on that formula: $N=Z^{2} x \quad p \quad x$ $\mathrm{q} / \mathrm{d}^{2}=322.7$ women $\quad(323$ women), $\mathrm{Z}$ : 1.96, p: $30 \%$, q: $1-0.30=0.70$, d: 0.05 ( $\mathrm{p}=15 \%$ in USA but in developing country can be twice or three $=30 \%$ ). But in seven months we recruited 324 participants and we excluded one participant.

Data analysis and interpretation were done by using Epi info 7, Microsoft excel spreadsheets for graphic confection and Microsoft word 2007 for text. Bivariate analysis was then conducted using chi square test to identify risk factors showing association with SSI. Means, standard deviation and tests of statistical significance were appropriately carried out and $\mathrm{P}$-values less than 0.05 were considered significant.

\section{Results and Discussion}

Three hundred twenty four pregnant women were enrolled in the study. Three hundred twenty three (99.7\%) were followed up completely for a total of 30 days post caesarean delivery. One woman enrolled died in the immediate postpartum period due to pulmonary embolism. The median age was 32 years and the prevalence of SSI in our study was $4.9 \%$. The majority of participants $(65.9 \%)$ were followed in our department $(12 / 23$ or $52.2 \%)$ compared to women with shorter labor $5 / 310$ or $1.6 \%(\mathrm{p}=<0.001)$. Most of SSI respondents' $(62.5 \%)$ was diagnosed in post discharge period. Significant association was found between prolonged $(>12 \mathrm{~h})$ labor and development of SSI $(3.4 \%) \quad(p=<0.0001)$ (RR: 29.7). Rupture membranes $(p=0.36)$, $\operatorname{ASA}(p=0.24)$, duration of surgery $(\mathrm{p}=0.83)$ and body mass index $(\mathrm{BMI})(\mathrm{p}=0.40)$ were not statistically significant in association with SSI development.

Caesarean Delivery (CD) was performed in $53.6 \%$ as an elective procedure. The most common indication of CD in our study was repeat caesarean $(52.6 \%)$ followed by fetal distress $(9.9 \%)$ and maternal request $(8.7 \%)$. This is a new phenomenon in obstetrics where the couple's decision was followed after informed consent and proper explanation of the surgical procedure and its complications. Caesarean delivery done as emergency had a two- fold increased risk of SSI rate than those of elective ones (3.4\%vs $1.5 \%)$

Regarding the microbes found in the pus culture, Staphylococcus aureus (62.5\%) was the most one found in SSI followed by Klebsiella pneumonia (12.5\%), Staphylococcus coagulase and Neisseria epidermidis with $6.25 \%$. Most of these organisms were sensitive to cephalosporin and quinolone. We did not encounter any methicillin resistant staphylococcus aureus (MRSA) in this sample. While simple drainage will usually suffice for superficial SSI, deep infections often required prolonged antibiotic treatment in addition to surgery in order to resolve the problem.

Concerning CDC type of SSI, Superficial SSI represented $75 \%$ in our study but deep SSI and organ SSI with $12.5 \%$ respectively. The majority of SSI were managed by wound opening, pus drainage and dressing twice daily but we encountered two cases with organ SSI where subtotal abdominal hysterectomy was required due to necrotized uterus, and two cases of necrotizing fasciitis requiring abdominal wall debridement. These women required prolonged hospitalization of more than 30 days for full recuperation. Most of SSI respondents' $(62.5 \%)$ was diagnosed in post discharge period. 
Prophylactic antibiotics and abdominal and thigh pre preparation for surgery with alcohol firstly and secondly povidone were used in $100 \%$ of our study population. The literature has found that chlorhexidine prep is superior for prevention of SSI, and a switch to that agent may help result in a lower SSI rate at our institution. Subcuticular suture $(78.9 \%)$ represented the most type of skin closure with a nonsignificant association with SSI development $\left(\mathrm{p}=0.24, \mathrm{x}^{2}=0.55\right)$.

The prevalence of surgical site infection in our study was $4.9 \%$. This rate was lower than that found in other developing countries: $11.4 \%$ at Jimma hospital in Ethiopia; however that study included all obstetrical procedures. A SSI rate of $9.1 \%$ was found in Kano, Nigeria but in Ward et $a l$, in a multi-center collaborative study of SSI following cesarean section in UK reported an overall wound problem incidence of $13.6 \%$ (seroma, hematoma, dehiscence and infection) and a SSI of $8.9 \%$ range between $2.9 \%$ to $17.9 \%$. The authors used similar post discharge surveillance process but in our study an additional system of calling patient at $15^{\text {th }}$ and 30rd day was done and an explanation of all symptoms and signs of SSI by investigators.

Our rate was lower than which found in Brazi19.6\% and in Australia 17\% but our rate was however higher than $3.2 \%$ reported by Barbot F et al.

Our study prevalence of SSI was lower than that of some other sitesdue to implementation of infection prevention and control (IPC) strategies (IPC training of personnel, hand hygiene, surgical safety check list), which was carried out in collaboration with Imperial College London staff before beginning our study.

Nevertheless prolonged labor was found to be significantly associated with SSI development ( $\mathrm{p}<0.0001, \mathrm{RR}: 29.7)$ which was similar to results found by Mpogoro et $a l$., and Ezechi et al.

Most of SSI following cesarean deliveries (CD) in our study were elective procedures $(53.6 \%)$ with repeat CD $(52.6 \%)$ as the most common indication. In contrast, in other studies, emergency CD wasmore common than elective procedures: Srun $\mathrm{S}$ et $a l$., and Ghuman M, Deirdre Rohlomet, et $a l$., reported SSI to be largely associated with operations performed urgently during labor.

Similar results were found in Jimma Hospital in Ethiopia with the risk of SSI rate being three-fold higher with emergency operations as opposed to those than electively.

No statistically significant difference in type of skin closure was found in our study or in that of Ibrahim et al.

SSI was confirmed by microbiological cultures in $87.5 \%$ of cases while $12.5 \%$ had negative cultures. These presumably may have represented anaerobic organism, difficult to isolate in our setting. Staphylococcus aureus was isolated in $62.5 \%$ of all cultures. This bacteria was shown to be the predominant organism in post CD SSI in our study, but others such as Klebsiella pneumonia (12.5\%), Staphylococcus coagulase negative, and Neisseria epidermidis with $6.25 \%$ each were also found. Most of these organisms were sensitive to a first or second generation of cephalosporin or a quinolone, as has been observed by others such as Jido et al., and Filbert J, Mpogoro et al.

Superficial SSI constituted the majority of SSI in our study at $75 \%$. This is similar to studies done by Chu et al., Muchini K., and Filbert J Mpogoro et al. 
Table.1 Epidemiological and clinical characteristics of participants $(\mathrm{N}=323)$ and SSI $(\mathrm{N}=16)$

\begin{tabular}{|c|c|c|c|c|}
\hline \multicolumn{2}{|l|}{ Characteristics } & \multirow[t]{2}{*}{ Frequency } & \multicolumn{2}{|c|}{$\begin{array}{lc}\text { Percentage(\%) } & \mathbf{p} \\
\text { No Infection/lnfectionvalue }\end{array}$} \\
\hline \multirow[t]{4}{*}{ Age (in years): } & Less than 20 & & $\begin{array}{l}2(0.6 \%) / 0 \\
(0 \%)\end{array}$ & \multirow{4}{*}{0.38} \\
\hline & $21-30$ & 127 & $121(37.4 \%) / 6(1.8 \%)$ & \\
\hline & $31-40$ & 178 & $168(52.0 \%) / 10(3.1 \%)$ & \\
\hline & $\geq 41$ & 16 & $16(4.9 \%) / 0(0 \%)$ & \\
\hline \multirow[t]{2}{*}{ Provenance } & Referred & 100 & $\begin{array}{l}94(29.1 \%) / \\
6(1.8 \%)\end{array}$ & \multirow{2}{*}{0.17} \\
\hline & Followed at CHUB & 223 & $213(65.9 \%) / 10(3.1 \%)$ & \\
\hline \multirow{2}{*}{ Body mass index } & $<30$ & 237 & $224(69.3 \%) / \mathbf{1 3}(4.0 \%)$ & \multirow{2}{*}{0.40} \\
\hline & $\geq 30$ & 86 & $83(25.7 \%) / 3(0.9 \%)$ & \\
\hline \multirow[t]{2}{*}{ Prolonged labor } & $\leq 12$ hours & 310 & $305(94.4 \%) / 5(1.5 \%)$ & \multirow{2}{*}{$\begin{array}{l}<0.0001 \\
\text { RR: } 29.7\end{array}$} \\
\hline & $>12$ hours & 23 & $12(3.7 \%) / \mathbf{1 1}(3.4 \%)$ & \\
\hline \multirow[t]{2}{*}{ Rupture of membranes } & $<18$ hours & 319 & $\begin{array}{l}305(94.4 \%) / \\
\mathbf{1 4}(4.3 \%)\end{array}$ & \multirow[t]{2}{*}{0.36} \\
\hline & $\geq 18$ hours & 4 & $\begin{array}{l}2(0.6 \%) / \\
2(0.6 \%)\end{array}$ & \\
\hline \multirow[t]{4}{*}{ Indication of Caesarean } & Repeat Caesarean & 175 & $\begin{array}{l}170(52.6 \%) / \\
5(1.5 \%)\end{array}$ & \multirow{4}{*}{0.26} \\
\hline & Foetal distress & 36 & $\begin{array}{l}32(9.9 \%) / \\
4(1.2 \%)\end{array}$ & \\
\hline & Maternal request & 30 & $\begin{array}{l}28(8.7 \%) / \\
2(0.6 \%)\end{array}$ & \\
\hline & Others & 82 & $\begin{array}{l}\mathbf{7 7}(23.8 \%) / \\
\mathbf{5 ( 1 . 5 \% )}\end{array}$ & \\
\hline \multirow[t]{2}{*}{ Caesarean category } & Emergency & 145 & $\begin{array}{l}\text { 134(41.5\%)/ } \\
\mathbf{1 1 ( 3 . 4 \% )}\end{array}$ & \\
\hline & Elective & 178 & $\begin{array}{l}173(53.6 \%) / \\
5(1.5 \%)\end{array}$ & \\
\hline \multirow[t]{2}{*}{ Skin closure } & Subcuticular & 268 & $\begin{array}{l}255(78.9 \%) / \\
13(4.0 \%)\end{array}$ & \multirow[b]{2}{*}{0.24} \\
\hline & Mattress & 55 & $\begin{array}{l}52(16.1 \%) / \\
\mathbf{3}(0.9 \%)\end{array}$ & \\
\hline \multirow{5}{*}{$\begin{array}{l}\text { Organism isolated } \\
\text { in the pus culture } \\
n=16\end{array}$} & Staphylococcus aureus & 10 & $62.5 \%$ & \\
\hline & Staphylococcus coagulase & 1 & $6.25 \%$ & \\
\hline & Klebsiella pneumonia & 2 & $12.5 \%$ & \\
\hline & Neisseria epidermis & 1 & $6.25 \%$ & \\
\hline & No bacteria & 2 & $12.5 \%$ & \\
\hline \multirow[t]{4}{*}{ ASA score } & I & 283 & 271(83.9\%)/12(3.7\%) & \multirow{4}{*}{0.24} \\
\hline & II & 34 & $31(9.6 \%) / 3(0.9 \%)$ & \\
\hline & III & 6 & $5(1.5 \%) / 1(0.3 \%)$ & \\
\hline & $\mathrm{IV} \& \mathrm{~V}$ & 0 & 0 & \\
\hline \multirow{3}{*}{$\begin{array}{l}\text { SSI } \\
\mathrm{n}=16\end{array}$} & Superficial & 12 & $75 \%$ & \\
\hline & Deep & 2 & $12.5 \%$ & \\
\hline & Organ & 2 & $12.5 \%$ & \\
\hline \multirow{2}{*}{$\begin{array}{l}\text { Time of SSI diagnosis } \\
\mathrm{n}=16\end{array}$} & Pre-discharge & 6 & $37.5 \%$ & \\
\hline & Post- discharge & 10 & $62.5 \%$ & \\
\hline
\end{tabular}


Most SSI were managed by wound opening, pus drainage, dressing twice daily and antibiotics, based on sensitivity found. As noted however, there were four complicated cases, two with peritonitis and necrotic uterus requiring hysterectomy, and two with necrotizing fasciitis requiring extensive abdominal wall debridement. These cases required prolonged antibiotic therapy and an extended hospital stay. Post CD SSI may become a life-threatening problem, emphasizing the need for good judgment prior to undertaking the procedure. Fortunately, no maternal mortality occurred in our sample.

In our study majority $(62.5 \%)$ of SSI infections were detected after discharge from the hospital. This may be explained by the short hospital stay after cesarean delivery. Most of SSI was identified within 15 days post cesarean delivery. There was a peak of infection noted between day 1 to 7 $(56.3 \%)$ after discharge from the hospital (mean day:1.3) but $31.3 \%$ of cases were found within day 8 to 15 (mean day:2.6) and $6.3 \%$ of cases were detected after 15 days.

Routinely in our department, all pregnant women underwent $\mathrm{CD}$ were discharged on the second post-operative day and the same results were found in previous studies: Conroy et al., Muchiri, 2014.

Use of mobile telephone in post discharge surveillance was found to be a very effective way to follow up patients in our setting. Two calls were made either directly to the patient, or through a relative or neighbor, by the study coordinator. An explanation of the symptoms and signs of was given by investigators (nurses) or study coordinator prior to discharge. This proved to be a very effective method of SSI monitoring, and post discharge adherence of respondents was able to reach $100 \%$. The cell phone is an instrument of modern technology that has penetrated worldwide, and has proved to be exceptionally helpful in our setting where most women live in locations remote from hospital. Similar findings were reported by Aiken et al., Bianeo et al., Muchini Kabau.

In conclusion, this study found a prevalence of SSI of $4.9 \%$ at Butare University Teaching Hospital (CHUB). Most of infections $(62.5 \%)$ developed after discharge and the majority were superficial surgical site infection $(75 \%)$ which were detected in post discharge surveillance within thirty days. The use of mobile telephone in post discharge surveillance with two contact calls proved to be an effective method of SSI monitoring after discharge from the hospital. Prolonged labor was identified as the most significant risk factor for SSI.

Therefore, prolonged labor as an important risk factor of SSI should be prevented through early intervention in case of protracted labor pattern. And establishment of post-operative visit at day 4 after discharge from the hospital, it will be a reasonable measure in SSI post discharge surveillance.

\section{List of Abbreviations}

-CHUB: Butare University Teaching Hospital

-CD: Cesarean Delivery

-SSI: Surgical Site Infection

-IPC: Infection Prevention and Control

-CDC: Center for Diseases and control

-BMI: Body Mass Index

-ASA: American Society for Anesthesiologist risk assessment tool

-CMHS-IRB: College of Medicine and Health Sciences Institutional Review Board

Ethical approval was given by the Butare University Teaching Hospital scientific and research committee and College of Medicine and Health Sciences Institutional Review Board(CMHS-IRB) 


\section{Authors' Contribution}

1. J.Kalibushi Bizimana: made substantial contributions to conception and design, or acquisition of data, or analysis and interpretation of data and drafting the manuscript

2. J. Ndoli Minega: Acquisition of data and have been involved in drafting the manuscript or revising it critically for important intellectual content;

3. C. Bayingana: Acquisition of laboratory data and have been involved in drafting the manuscript or revising it critically for important intellectual content;

4. J.G. Gilson: made substantial contributions to conception and design, or acquisition of data and interpretation of data and drafting the manuscript

5. I. Baluhe: Acquisition of data, participants follow up and agreed to be accountable for all aspects of the work in ensuring that questions related to the accuracy or integrity of any part of the work are appropriately investigated and resolved.

6. E. Habimana: Acquisition of data and have been involved in drafting the manuscript or revising it critically for important intellectual content

\section{Acknowledgements}

This study could not have been completed without the cooperation of multiple institutional personnel. We would like to acknowledge CHUB for financing and logistic support, and allowing access in patients' files. Our appreciation also goes to all Obstetrician and Gynecologists, Residents, Midwives and Nurses at CHUB/ObGyn for their valuable contributions and especially those who participated in data collection. Finally but not the least, we thank all of you who contributed for the accomplishment of this study in one way or other and you are not mentioned above, we extend our sincere thanks and profound appreciation

\section{References}

Aiken, A.M. et al. 2013. Evaluation of surveillance for surgical site infections in Thika Hospital Kenya. J. Hosp., 83: 140-145.

Arabashahi, K.S., Koohpayezade, J. 2006. Investigation of risk factors for surgical wound infection among teaching hospital in Tehran. Int. Wound J., 3: 59-62. doi: 10.1111/j.1742-4801.2006.00186e.x.

Barbot, F., Carbonne, B., Truchot, F., Spielvogel, C., Jannet, D., Goderel, I., et al. 2004. Surgical site infections after cesarean section: Results of a five-year prospective surveillance. $J$. Gynecol. Obstet. Biol. Reprod., (Paris) 33: 487-96.

Beigi, R., Hnrahan, J. 2007. Staphylococcus aureus and MRSA colonization rate among Gravidas admitted to labor and delivery: Apilot study. Infect. Dis. Obstet. Gynecol., Volume (70876) 4pages

Bianco, A., et al. 2013. Post discharge surveillance following delivery: the incidence of infections and associated factors. Am. J. infect. Control, 41: 542-543.

Brasil. Prefeitura de Belo Horizonte. Secretaria Municipal de Saúde. Gerênciade VigilânciaemSaúde e Informação. Boletim de Vigilância $e$ Saúde, 1(10): 5-7.

CDC/NHSN Surveillance definition of healthcare-associated infection and criteria for specific types of infection in the acute care setting. 2013. Centers for Disease Control and Prevention.http://www.cdc.gov/nhsn/P 
DFs/pscManual/17pscNosInfDef_curr ent.pdf

Couto, R.C., Pedrosa, T.M., Nogueira, J.M., et al. 1998. Post discharge surveillance and infection rates in obstetric patients. Int. J. Gynaecol. Obstet., 61(3): 227-231. doi:10.1016/S00207292(98)00047-2

Cunningham, F.G., Leveno, K.J., Bloom, SL.., Hanth, J.C., Gilstrap, L,. Wensrom, K.D. Williams Obstetrics $22^{\text {nd }}$ edition.

Cuver, D.H., Horan, TC., Gynes, R.P. et al. 1991. Surgical wound infection rates by wound class, procedure, and patient risk index NNIS system. Am. J. Med., 91(suppl B): 152-157.

Danforth's Obstetrics and gynecology Tenth edition chapter 27, 2008pg 491

Demisew, A., Tefera, Fitsum Araya. 2010. Surgical Site Infection and risk factors among Obsteric cases of Jimma Univerity Specialized Hospital, Southwest Ethiopia April 2009 to March 2010; Ethiop. J. Health Sci., 21(2): 91-100.

Diluigi, A. et al. 2004. Prophylactic antibiotic administration prior to hysterectomy: A quality improvement initiative. J. Repro health med., 49(12): 949-954.

Ghuman, M., et al. 2011. Post-caesarean section surgical site infection: rate and risk factors. N. Z. Med, J., 124 (1339): 32-6.

Hulton, L.J., Olmsted, R.N., Trestin-Aurand, J., et al. Effect of post discharge surveillance on rates of infections and complications after caesarean section. Am. J. Infect. Control, 20: 198e201.

Ibrahim, M.I. et al. 2014. Superficial incisional surgical site infection rate after cesarean section in obese women: a randomized controlled trial of subcuticular versus interrupted skin suturing. Arch. Gynecol. Obstet., 289(5): 981-6. doi:10.1007

Jido, T.A., Garba, I.D. 2012. Surgical-site infection following cesarean section in Kano, Nigeria. Ann Med Health Sci Res [serial online] [cited 2015 Sep 14]; 2: 33-6.

John Larsen. W. 2003. Guidelines for the diagnosis, treatment and prevention of postoperative infection. Infect. Dis. Obstet. Gynecol., 11: 65-70.

Kathryn, Chu, R., Maine, M. Trelles. 2014. Cesarean section surgical site infection in sub-Saharan Africa: Amulti-country study from Medecins Sans Frontieres. World J. Surg., 39: 350-355. Published online 2014 Oct 31, doi: 10.1007/s00268-014-28404

Kelly Conroy, et al. 2012. Infectious morbidity after cesarean delivery: 10 strategies to reduce the risk, Rev. Obstet. Gynecol., 5(2): 69-77.

Mpogoro, F.J et al. 2014. Incidence and predictors of surgical site infections following caesarean sections at Bugando Medical Centre, Mwanza, Tanzania Bio Medical Centre open access.

Muchiri Kabau, D. 2014. Incidence and determinants of surgical site infection after caesarean delivery at Kenyatta National Hospital 2014Mmed dissertation.

Oliver, C., Ezechi, et al. 2009. Incidence and risk factors for caesarean wound infection in Lagos Nigeria. BMC research note.

Petroze, R.T., Nzayisenga, A., Calland, J.F., Ntakiyiruta, G. Surgical Volumes at the District Hospital: A Retrospective Review of National Data in Rwanda East and Central African J. Surgery, Vol. 16, No. 2, July/August, 2011, pp. 102-111.

Plowman, R., Graves, N., Griffin, M. 2001. The rate and cost of hospital-acquired 
infections occurring in patients admitted to selected specialities of a district general hospital in England and the national burden imposed. $J$. Hosp. Infect., 47: 198e209.

Protocol for surveillance of surgical site infection, surgical site infection service version 6 Public Health England June 2013.

Raka, L., Zounman, D. 2006. Prevalence of nosocomial infections in high risk units in the University clinical center of Kosovo. Infect. Control and Hospital Epidemiol., 27(4): 421-423.

Robert, M., Michael, P., Berry Robert, E., et al. 1993. Avoiding serious infections associated with abdominal hysterectomy: A meta-analysis of antibiotic prophylaxis. Clin. American J. Obstetrics and Gynecol., 169(5): 1119-1124.

Rock John, A., Jones Howard, W., Te Linde's operative gynecology $10^{\text {th }}$ edition 2008.

SokSrun, et al. 2013. Surveillance of post cesarean section surgical site infections in a hospital with limited resources, Colombia J. Infect. Dev. Ctries., 7(8): 579-585.

Torre, S., Mandel, L., Goff, B. Evaluation of post-operative fever: Usefulness and cost-effectiveness of routine workup. American J. Obstetrics and Gynecol., volume 188 , issue 6 , page $1642-1647$

Tran, Thach, S., et al. 2000. Risk factors for post cesarean surgical site infection. Obstetrics and Gynecol., volume 95, page 367-371.

Ward, V.P., Charlett, A., Fagan, J., Crawshaw, S.C. 2008. Enhanced surgical site infection surveillance following caesarean section: Experience of a multicenter collaborative post-discharge system. $J$. Hosp. Infect., 70: 166-73.

WHO. Division of health family. The prevention and management of puerperal infections: Report of a technical working group, Geneva 2022 May

1992-1995.

\section{How to cite this article:}

J. Kalibushi Bizimana, J. Ndoli, C.Bayingana, I.Baluhe, G.J.Gilson and E.Habimana. 2016. Prevalence and Risk Factors for Post Cesarean Delivery Surgical Site Infection in a Teaching Hospital Setting in Rural Rwanda: A Prospective Cross Sectional Study. Int.J.Curr.Microbiol.App.Sci. 5(6): 631-641. doi: http://dx.doi.org/10.20546/ijcmas.2016.506.069 